\title{
SDSS-IV MaNGA: Variation of the Stellar Initial Mass Function in Spiral and Early-type Galaxies
}

\author{
Hongyu $\mathrm{Li}^{1,2}$, Junqiang $\mathrm{Ge}^{1}$, Shude Mao ${ }^{1,3,4}$, Michele Cappellari ${ }^{5}$, R. J. Long ${ }^{1,4}$, ${\text { Ran } \mathrm{Li}^{1,6} \text {, Eric Emsellem }}^{7,8}$, Aaron A. Dutton ${ }^{9}$, \\ Cheng $\mathrm{Li}^{3,10}$, Kevin Bundy ${ }^{11}$, Daniel Thomas ${ }^{12}$, Niv Drory ${ }^{13}$, and Alexandre Roman Lopes ${ }^{14}$ \\ ${ }^{1}$ National Astronomical Observatories, Chinese Academy of Sciences, 20A Datun Road, Chaoyang District, Beijing 100012, China; hyli@nao.cas.cn \\ ${ }^{2}$ University of Chinese Academy of Sciences, Beijing 100049, China \\ ${ }^{3}$ Physics Department and Tsinghua Centre for Astrophysics, Tsinghua University, Beijing 100084, China \\ ${ }^{4}$ Jodrell Bank Centre for Astrophysics, School of Physics and Astronomy, The University of Manchester, Oxford Road, Manchester M13 9PL, UK \\ ${ }^{5}$ Sub-Department of Astrophysics, Department of Physics, University of Oxford, Denys Wilkinson Building, Keble Road, Oxford, OX1 3RH, UK \\ ${ }^{6}$ Key laboratory for Computational Astrophysics, National Astronomical Observatories, Chinese Academy of Sciences, Beijing, 100012, China \\ ${ }^{7}$ Université Lyon 1, Observatoire de Lyon, Centre de Recherche Astrophysique de Lyon and Ecole Normale Supérieure de Lyon, 9 avenue Charles André, \\ F-69230 Saint-Genis Laval, France \\ ${ }^{8}$ European Southern Observatory, Karl-Schwarzschild-Str. 2, D-85748 Garching, Germany \\ ${ }^{9}$ New York University Abu Dhabi, P.O. Box 129188, Abu Dhabi, UAE \\ ${ }^{10}$ Shanghai Astronomical Observatory, Shanghai 200030, China \\ ${ }^{11}$ Kavli IPMU (WPI), UTIAS, The University of Tokyo, Kashiwa, Chiba 277-8583, Japan \\ 12 Institute of Cosmology \& Gravitation, University of Portsmouth, Dennis Sciama Building, Portsmouth, PO1 3FX, UK \\ ${ }^{13}$ McDonald Observatory, The University of Texas at Austin, 1 University Station, Austin, TX 78712, USA \\ ${ }^{14}$ Department of Physics and Astronomy, Universidad de La Serena, Cisternas 1200, La Serena, Chile \\ Received 2016 August 11; revised 2017 February 24; accepted 2017 March 9; published 2017 March 29
}

\begin{abstract}
We perform Jeans anisotropic modeling (JAM) on elliptical and spiral galaxies from the MaNGA DR13 sample. By comparing the stellar mass-to-light ratios estimated from stellar population synthesis and from JAM, we find a systematic variation of the initial mass function (IMF) similar to that in the earlier ATLAS $^{3 \mathrm{D}}$ results. Early-type galaxies (elliptical and lenticular) with lower velocity dispersions within one effective radius are consistent with a Chabrier-like IMF, while galaxies with higher velocity dispersions are consistent with a more bottom-heavy IMF such as the Salpeter IMF. Spiral galaxies have similar systematic IMF variations, but with slightly different slopes and larger scatters, due to the uncertainties caused by the higher gas fractions and extinctions for these galaxies. Furthermore, we examine the effects of stellar mass-to-light ratio gradients on our JAM modeling, and we find that the trends become stronger after considering the gradients.
\end{abstract}

Key words: dark matter - galaxies: evolution - galaxies: formation - galaxies: kinematics and dynamics galaxies: structure

\section{Introduction}

Stellar mass is one of the fundamental attributes of a galaxy. Accurate estimation of stellar mass plays an important role in the study of a galaxy's structure, evolution, and formation (Cappellari 2016). Stellar population synthesis (SPS) is the most popular method for obtaining the stellar mass. However, the stellar mass so obtained depends strongly on assuming a stellar initial mass function (IMF). Estimated stellar masses will shift on average 0.25 dex higher by changing the IMF from the Chabrier IMF (Chabrier 2003) to the Salpeter IMF (Salpeter 1955) (see Panter et al. 2007; Tortora et al. 2009). This causes uncertainty in the determination of the dark matter fraction in a galaxy's central region (Cappellari et al. 2006; Tortora et al. 2009). Furthermore, whether the IMF is universal or not has been discussed for decades (Bastian et al. 2010). The situation is becoming clearer after numerous studies were conducted based on line-strength indices (e.g., Conroy \& van Dokkum 2012; Spiniello et al. 2012), strong lensing plus spatially unresolved stellar kinematics (e.g., Treu et al. 2010; Posacki et al. 2015), resolved stellar kinematics (Cappellari et al. 2012, 2013a), and the fundamental plane (Dutton et al. 2013a). All these studies show evidence for variation of the IMF in early-type (i.e., elliptical or lenticular) galaxies.

In Cappellari et al. (2012), the Jeans anisotropic modeling technique (JAM; Cappellari 2008) was used, with a spatially constant stellar mass-to-light ratio and several different dark matter halo models, to obtain stellar mass estimates without resorting to SPS. They used 256 early-type galaxies from the ATLAS $^{3 \mathrm{D}}$ integral field unit (IFU) survey and found a systematic variation in the IMF with galaxy stellar mass-tolight ratio. With the increasing availability of IFUs, more and more nearby galaxies with IFU data are becoming available, for example, CALIFA (Sánchez et al. 2012), SAMI (Bryant et al. 2015), and MaNGA (Bundy et al. 2015). The MaNGA DR13 (SDSS Collaboration et al. 2016) sample includes 1390 galaxies of different morphologies (both early- and late-type galaxies) and is currently the largest IFU sample. Please see the following references for more details about the MaNGA instrumentation (Drory et al. 2015), observing strategy (Law et al. 2015), spectrophotometric calibration (Smee et al. 2013; Yan et al. 2016a), and survey execution and initial data quality (Yan et al. 2016b). In this paper, we take advantage of the MaNGA DR13 sample to investigate IMF variation for both early-type and late-type galaxies using a method similar to that of Cappellari et al. (2012). Furthermore, we examine the effects of stellar mass-to-light ratio gradients (Portinari \& Salucci 2010; Tortora et al. 2011; J. Ge et al. 2017, in preparation) on our dynamical models (i.e., not using a constant stellar mass-to-light ratio).

The structure of this paper is as follows. In Section 2, we briefly introduce the galaxy sample and the modeling methods 
we use. In Section 3, we show our results concerning the systematic variation of the IMF and the effects of stellar massto-light ratio gradients. In Section 4, we summarize and give our conclusions.

\section{MaNGA Sample and Methods}

\subsection{MaNGA Sample}

We use the MaNGA Product Launch 4 (MPL4) IFU spectra from the MaNGA DR13 SDSS-IV sample. The IFU spectra are extracted using the official data reduction pipeline (Law et al. 2016), and kinematical data are extracted using the official data analysis pipeline (K. Westfall et al. 2017, in preparation). From the 1390 galaxies in the MaNGA DR13 catalog, we exclude merging galaxies (close galaxy pairs, extremely unsmooth structures) and galaxies with low data quality (with less than 20 Voronoi bins with signal-to-noise $(\mathrm{S} / \mathrm{N})$ greater than 30$)$. In total, we are left with 816 galaxies (413 spiral galaxies; 403 elliptical and lenticular galaxies), more than a factor of three times larger than the ATLAS ${ }^{3 D}$ sample. We visually select galaxies with the best data qualities and JAM modeling as our class A subsample (sufficient Voronoi bins, high S/N, no strong bars and spiral arms- these galaxies will have reliable JAM models). There are 346 galaxies in the class A subsample. We match our whole galaxy sample with Galaxy Zoo 1 (Lintott et al. 2008) to obtain galaxy morphologies.

\subsection{Stellar Population Synthesis}

To asses the robustness of our results, we derive the stellar masses of all our galaxies using two different full spectrum fitting software packages and two different SPS template libraries. In both software packages, we adopted for reference a Salpeter (1955) IMF. The first stellar mass estimate uses the STARLIGHT software (Cid Fernandes et al. 2005), in combination with the BC03 SPS templates (Bruzual \& Charlot 2003), while the second uses the pPXF software (Cappellari \& Emsellem 2004; Cappellari 2017) with the MILES-based (Sánchez-Blázquez et al. 2006) SPS models of Vazdekis et al. (2010). For STARLIGHT, we use 25 ages $(\log$ Age $=[6.00,6.50,6.70,6.82,6.94,7.00,7.16,7.40,7.60$, $7.74,8.01,8.21,8.46,8.71,8.96,9.11,9.16,9.40,9.63,9.80$, $9.88,10.00,10.11,10.18,10.26]$ years) and six metallicities $([\mathrm{Z} / \mathrm{H}]=[-1.7,-1.3,-0.7,-0.4,0.0,0.4])$. For pPXF, we use 25 ages linearly spaced in logAge (years) between 7.8 and 10.25 and six metallicities $([\mathrm{Z} / \mathrm{H}]=[-1.7,-1.3,-0.7,-0.4$, $0.0,0.2])$. As can be seen from Figure 1, the stellar mass-tolight ratios obtained from different templates and software agree well for galaxies with higher stellar mass-to-light ratios (i.e., old ages). The scatter is less than $0.1 \mathrm{dex}$ and has nearly no bias. For galaxies with lower stellar mass-tolight ratios (i.e., young ages), pPXF with MILES templates gives systematically higher stellar mass-to-light ratios than STARLIGHT with $\mathrm{BC} 03$. This is because the $M^{*} / L$ is more degenerate for younger galaxies than for older ones. The reason for this is that in young galaxies the light of a few bright stars can dominate the flux in a galaxy's spectrum. This makes it easy to "hide" significant numbers of old stars, which emit a small amount of light but contribute significantly to the mass, increasing the $M^{*} / L$. However, the difference between the Salpeter and Chabrier IMF is $0.25 \mathrm{dex}$, so this $0.1 \mathrm{dex}$ difference will not strongly affect our conclusions. This is

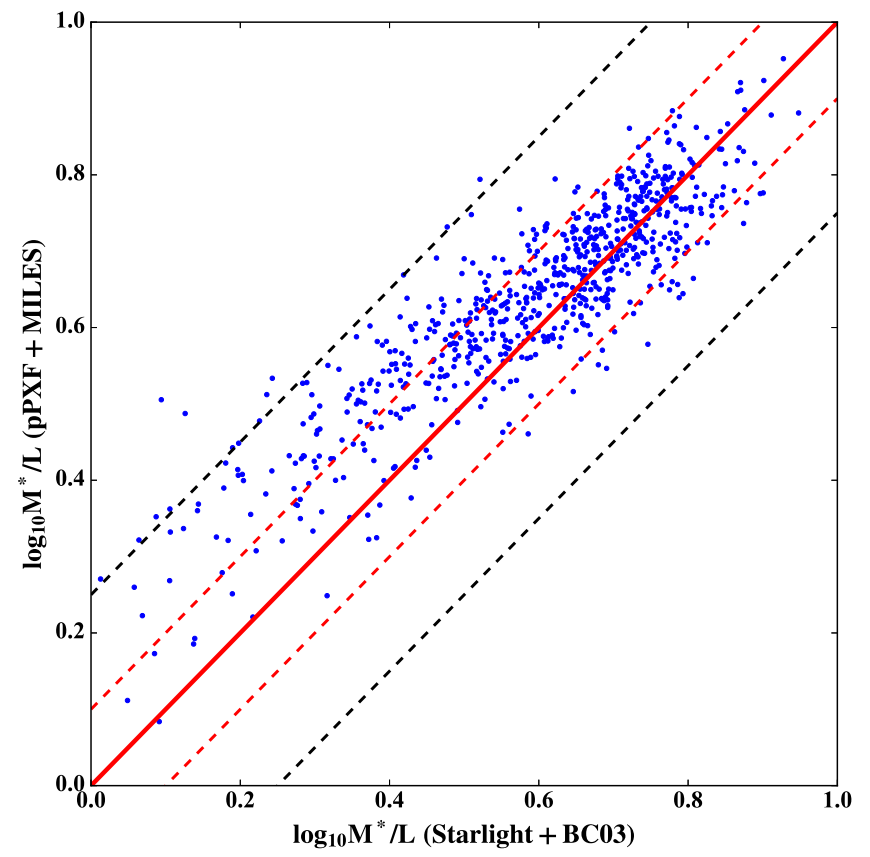

Figure 1. Comparison of the stellar mass-to-light ratios for all 816 galaxies between the pPXF software with MILES templates and the STARLIGHT software with BC03 templates. The red dashed lines show a 0.1 dex difference, and the black dashed lines show a 0.25 dex difference, which is the difference between the Salpeter and Chabrier IMFs.

shown explicitly in Section 3.1, where consistent IMF trends are presented using stellar masses from both STARLIGHT and pPXF. We use 0.1 dex as the uncertainty in the SPS in the following analysis. More details about the comparison of software packages and templates can be found in J. Ge et al. (2017, in preparation).

We calculate our stellar mass-to-light ratios using Equation (2) in Cappellari et al. (2013a):

$$
\left(M^{*} / L\right)_{\mathrm{SPS}}=\frac{\sum_{\mathrm{j}=1}^{\mathrm{N}} w_{\mathrm{j}} M_{\mathrm{j}}^{\text {nogas }}}{\sum_{\mathrm{j}=1}^{\mathrm{N}} w_{\mathrm{j}} L_{\mathrm{j}}},
$$

where $M_{\mathrm{j}}^{\text {nogas }}$ is the stellar mass of the $j$ th template, which includes the mass in living stars and stellar remnants, but excludes the gas lost during stellar evolution; $L_{\mathrm{j}}$ is the corresponding r-band luminosity; $w_{\mathrm{j}}$ is the weight of the $j$ th template.

Before spectrum fitting, the data cubes are Voronoi binned (Cappellari \& Copin 2003) to an $\mathrm{S} / \mathrm{N}=30$. For all resulting Voronoi bins in each galaxy, the two softwares fit for both of the template weights and for the dust extinction, adopting a Calzetti et al. (2000) reddening curve. The luminosity of each spatial bin is separately corrected for the measured extinction, before computing the total r-band $\left(M^{*} / L\right)_{\text {SPS }}$ for a galaxy by summing the luminosity and masses of all the bins within the MaNGA field of view. This dust extinction corrected $\left(M^{*} / L\right)_{\text {SPS }}$ is directly comparable with the stellar mass-to-light ratios in JAM modeling, obtained from stellar masses divided by the observed r-band luminosities. See Section 3.3 for more discussion about the extinction and inclination effects. 


\subsection{Dynamical Modeling}

We perform JAM (Cappellari 2008) for all 816 selected galaxies. For a given luminosity density (constructed by fitting a galaxy's surface brightness using mge_fit_sectors software (Cappellari 2002) and deprojecting it using the Multi-Gaussian Expansion (MGE) method of Emsellem et al. 1994), we assume a spatially constant stellar mass-to-light ratio and a gNFW dark halo profile (also see Barnabè et al. 2012; Cappellari et al. 2013a)

$$
\rho_{\mathrm{DM}}(r)=\rho_{s}\left(\frac{r}{R_{s}}\right)^{\gamma}\left(\frac{1}{2}+\frac{1}{2} \frac{r}{R_{s}}\right)^{-\gamma-3}
$$

to construct a galaxy's total mass model. From running JAM within a Markov chain Monte Carlo (MCMC) framework (Foreman-Mackey et al. 2013), we find the best-fitting parameters (including the stellar mass-to-light ratio $\left.\left(M^{*} / L\right)_{\mathrm{JAM}}\right)$ that give the best model matching the galaxy's observed second velocity moment map. We correct the cosmological surface brightness dimming effect in our MGE by multiplying the surface brightness by a factor $(1+z)^{3}$, which accounts both for the bolometric surface brightness dimming and the change of the bandwidth (in the AB system). Since the MaNGA galaxies are mostly of low redshift (median and maximum redshifts of the MaNGA sample are 0.03 and 0.15 , respectively), we choose not to apply K-correction (e.g., Hogg et al. 2002) here. The stellar mass-to-light ratios obtained from this method are independent of SPS and so can be used to test the variation of the IMF. The details of the modeling process are described in $\mathrm{Li}$ et al. (2016), which assesses the validity of the JAM method using cosmologically simulated galaxies. We broaden the prior for the central dark halo slope $\gamma$ from $[-1.2,0]$ to $[-1.6,0]$ to avoid the bias in the IMF, which is sensitive to the halo response to baryonic settling (Dutton et al. 2013a). The prior is consistent with simulated halos in the EAGLE cosmological simulation (Schaller et al. 2015), as well as elliptical galaxy zoom-in simulations, which find inner slopes of $\sim-1.6$ (Dutton et al. 2015), and spiral galaxy simulations, which can have inner slopes of $\sim 0$ (Tollet et al. 2016).

Since spiral galaxies have higher gas fractions, especially later-type spirals (Huang et al. 2012; Combes et al. 2013; Jaskot et al. 2015), we need to consider the gas contribution to the stellar masses derived from JAM. We perform the following steps to reduce the effects of cold gas in spiral galaxies:

1. We use the $M^{\text {gas }}-M^{*}$ relation from Huang et al. (2012, Equation (1)) to estimate the gas mass for every spiral galaxy. The stellar masses we use in applying the Huang relationship are taken from SPS.

2. We assume the gas $\left(\mathrm{HI}+\mathrm{H}_{2}\right)$ mass distribution can be approximated by an exponential disk with scale length $6.1 \mathrm{kpc}$ (Bigiel \& Blitz 2012).

3 . We calculate the gas mass within $2.5 R_{\mathrm{e}}$ for every spiral galaxy using the gas mass profile described above.

4. We use the formula below to correct for the effect of gas in the JAM stellar mass-to-light ratios for spiral galaxies

$$
\left(M^{*} / L\right)_{\mathrm{JAM}}^{\mathrm{nogas}}=\frac{M_{\mathrm{JAM}}^{*}-M^{\text {gas }}\left(<2.5 R_{\mathrm{e}}\right)}{L},
$$

where $\left(M^{*} / L\right)_{\mathrm{JAM}}^{\mathrm{nogas}}$ is the final value that we use to

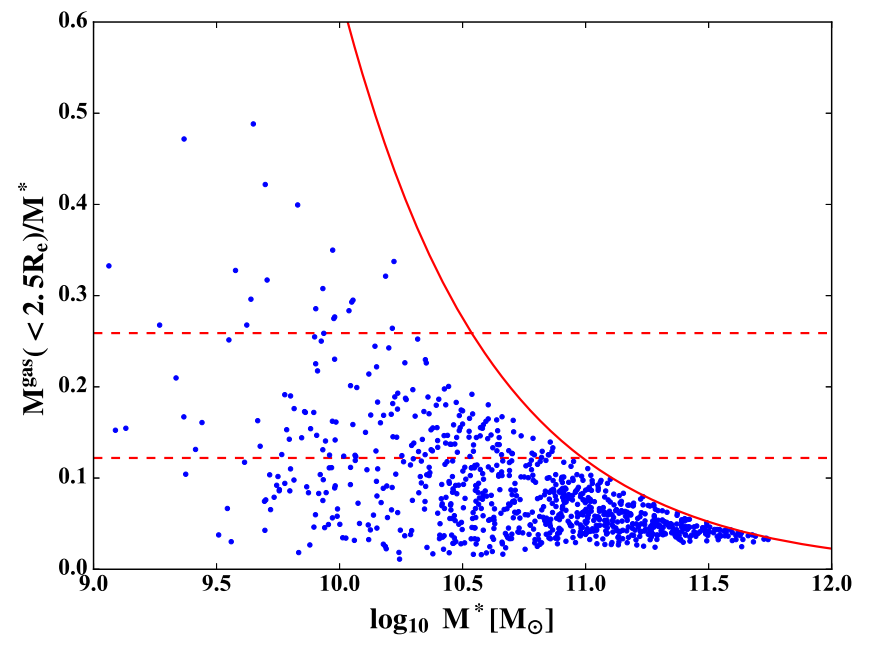

Figure 2. Gas mass fraction for all of the spiral galaxies. The red solid line shows the gas fraction for a given stellar mass if all of the gas is within $2.5 R_{\mathrm{e}}$. The red dashed lines show the fraction at which the $M^{*} / L_{\mathrm{JAM}}$ will change by 0.05 dex and 0.1 dex in the gas correction, respectively.

investigate IMFs, $M_{\mathrm{JAM}}^{*}$ is the stellar mass estimated by $\mathrm{JAM}$, and $M^{\text {gas }}\left(<2.5 R_{\mathrm{e}}\right)$ is the gas mass within $2.5 R_{\mathrm{e}}$.

In Figure 2, we plot the gas mass fraction within $2.5 R_{\mathrm{e}}$ versus galaxy stellar mass to show the impact of the gas correction. As can be seen, for massive spiral galaxies $\left(\log M^{*}>11.0\right)$, the gas fraction is smaller than $10 \%$, which has nearly no effect on the dynamical models. As shown by the red dashed lines, the change of $\left(M^{*} / L\right)_{\mathrm{JAM}}$ is smaller than $0.05 \mathrm{dex}$ for more than half of the galaxies, and smaller than 0.1 dex for more than $90 \%$ of the galaxies.

\section{Results}

In this section, we first show the systematic variation of the IMF and make a comparison with ATLAS ${ }^{3 \mathrm{D}}$ results. We then show the results from including the stellar mass-to-light ratio gradients.

\subsection{Systematic Variation of IMF}

In order to describe the variation of the IMF, we define the IMF mismatch parameter similar to Treu et al. (2010):

$$
\alpha_{\mathrm{IMF}} \equiv\left(M^{*} / L\right)_{\mathrm{JAM}}^{\text {nogas }} /\left(M^{*} / L\right)_{\mathrm{SPS}},
$$

where $\alpha_{\mathrm{IMF}}$ is the ratio of the $M^{*} / L$ values obtained by JAM and SPS for a Salpeter IMF. In Figure 3, we plot the mismatch parameter $\alpha_{\mathrm{IMF}}$ versus the velocity dispersion within an effective radius. The left panels are for the results from STARLIGHT + BC03, while the right panels are for the results from pPXF + MILES. The velocity dispersion is defined as

$$
\sigma_{\mathrm{e}}=\sqrt{\left\langle v_{\text {los }}^{2}\left(<R_{\mathrm{e}}\right)\right\rangle},
$$

with $v_{\text {los }}^{2} \equiv V^{2}+\sigma^{2}$, where $V$ and $\sigma$ are the mean velocity and dispersion of the Gaussian that best fits the line-of-sight velocity distribution. A parameter value of $\alpha_{\mathrm{IMF}}=1$ means that JAM and SPS give the same estimate.

As can be seen from the panels, $\alpha_{\mathrm{IMF}}$ changes systematically with velocity dispersion. Galaxies with higher velocity dispersions are consistent with a Salpeter IMF, while galaxies with lower velocity dispersions are consistent with a Chabrier 

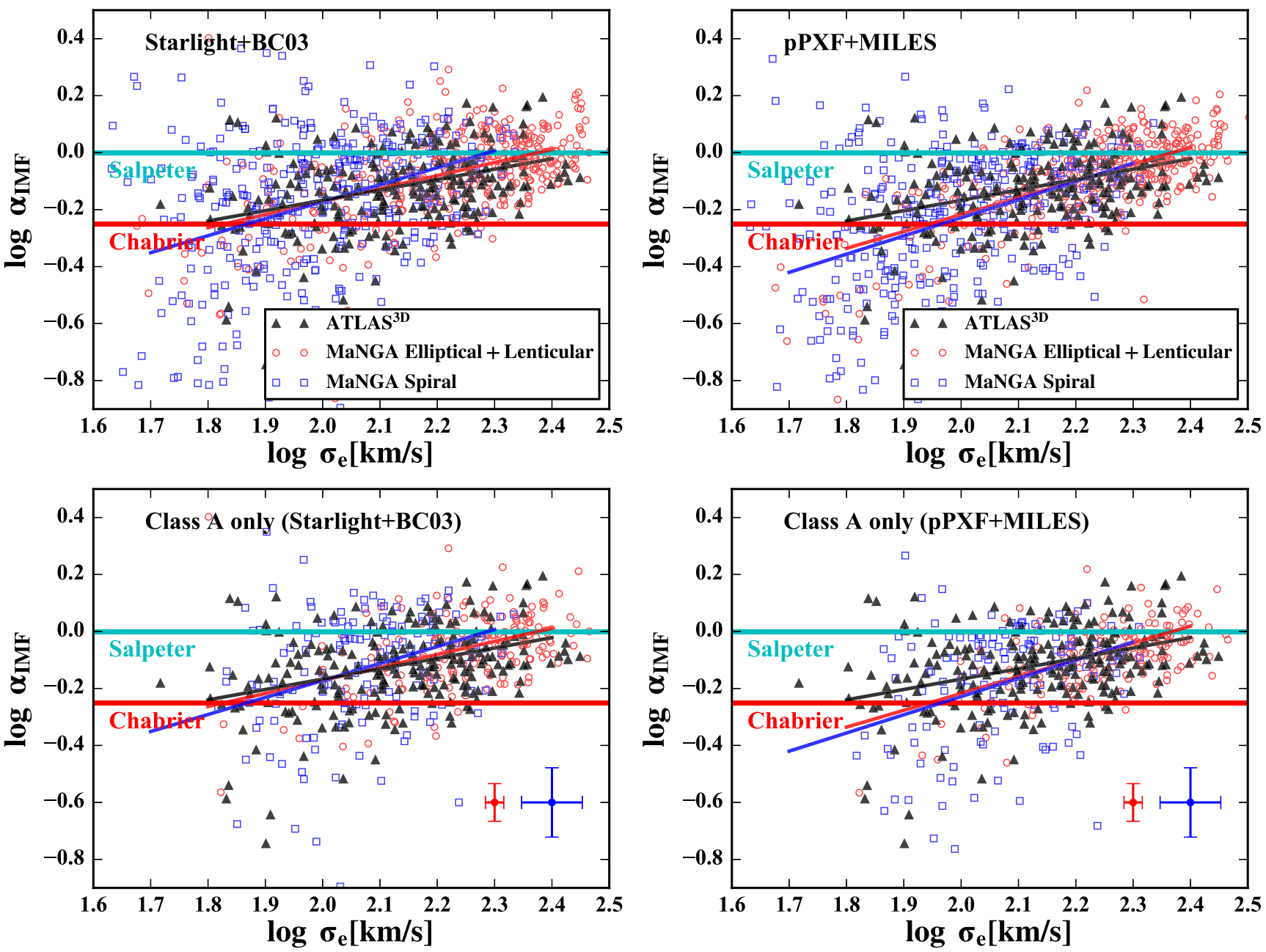

Figure 3. Systematic IMF variation for MaNGA galaxies. Upper left: the $\log \alpha_{\mathrm{IMF}}$ for STARLIGHT + BC03 vs. galaxy velocity dispersion. Lower left: class A sample only, which has the most reliable fitting among the whole sample. Upper right: the log $\alpha_{\mathrm{IMF}}$ for pPXF + MILES vs. galaxy velocity dispersion. Lower right: class A sample only. In each panel, the black triangles show the results from ATLAS ${ }^{3 \mathrm{D}}$, red circles for the MaNGA elliptical galaxies, and blue squares for MaNGA spiral galaxies. The solid lines show the linear fitting results respectively. The horizontal, colored solid lines show the positions where the stellar mass-to-light ratios from JAM equal the SPS with a Salpeter IMF (cyan) and the SPS with a Chabrier IMF (red). The mean errors for elliptical and spiral galaxies are shown in the red and blue error bars in the lower panels, respectively. The error in $\left(M^{*} / L\right)_{\mathrm{JAM}}$ is estimated using the MCMC 1D marginalized distribution, while the error in $\left(M^{*} / L\right)_{\mathrm{SPS}}$ is obtained by using different stellar templates and software in SPS.

IMF. This is true for both elliptical and spiral galaxies, although there are larger scatters for spiral galaxies due to the effects from cold gas, dust extinction, and larger degeneracies between dark matter and stellar mass.

We compare the systematic variation between different SPS software packages and templates in the left and right panels (left for STARLIGHT+BC03, right for pPXF+ MILES). As can be seen, there are some small differences between the two approaches, but the trends are consistent within the statistical errors, as quantified in Table 1 . The small differences can be understood as being due to the residual systematic differences between the two approaches, illustrated in Figure 1. We fit the trend using a linear relation

$$
\log \alpha_{\mathrm{IMF}}=a+b \times \log \sigma_{\mathrm{e}}
$$

The fitting results are listed in Table 1 and plotted in Figure 3.

The scatter for spiral galaxies with lower velocity dispersions is large since they are more affected by cold gas and dust extinction. The degeneracy between dark matter and stellar mass for these galaxies is also larger. There are some outliers with low velocity dispersion but high $\alpha_{\mathrm{IMF}}$. We check the JAM
Table 1

Fitting Coefficients of the $\log \sigma_{\mathrm{e}}-\log \alpha$ Relation (Equation (6)) for Elliptical and Spiral Galaxies

\begin{tabular}{lccc}
\hline \hline & $a$ & $b$ & $\Delta_{\text {int }}$ \\
\hline $\begin{array}{l}\text { MaNGA elliptical } \\
\quad \text { (STARLIGHT) }\end{array}$ & $-1.086 \pm 0.006$ & $0.457 \pm 0.033$ & 0.082 \\
MaNGA elliptical (pPXF) & $-1.399 \pm 0.005$ & $0.591 \pm 0.030$ & 0.063 \\
MaNGA spiral & $-1.364 \pm 0.011$ & $0.596 \pm 0.069$ & 0.156 \\
$\quad$ (STARLIGHT) & & & \\
MaNGA spiral (pPXF) $_{\text {ATLAS }}{ }^{\text {M }}$ & $-1.506 \pm 0.011$ & $0.638 \pm 0.075$ & 0.173 \\
& $-0.895 \pm 0.009$ & $0.364 \pm 0.042$ & 0.083 \\
\hline
\end{tabular}

Notes. The units of $\log \sigma_{\mathrm{e}}$ in the fitting are $\mathrm{km} \mathrm{s}^{-1} ; \Delta_{\text {int }}$ is the intrinsic scatter. The fitting is performed using the lts_linefit software from Cappellari et al. (2013b).

and SPS model fitting results for these galaxies, and we find that they are galaxies with poor data quality (large uncertainties in dynamical modeling and SPS) or high inclination (edge-on galaxies, strong dust extinction). Our results are also consistent with results from gravitational lensing, albeit at slightly higher 

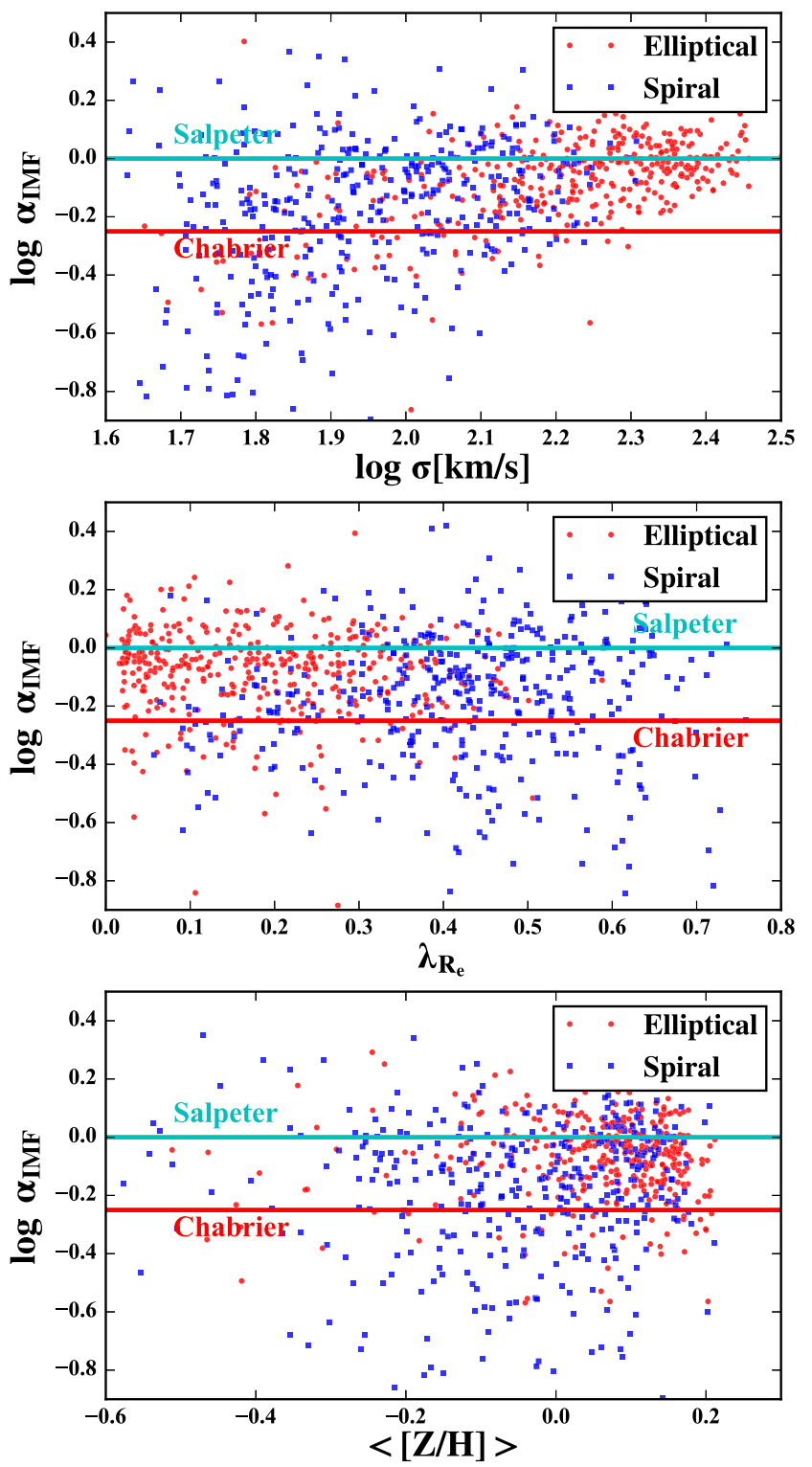

Figure 4. $\log \alpha_{\mathrm{IMF}}$ vs. $\log \sigma$ (top), $\lambda_{R_{\mathrm{e}}}$ (middle), and metallicity (bottom). Other labels and legends are the same as in Figure 3.

redshift $z=0.2$ (Treu et al. 2010). However, there are also a few discordant cases in Smith \& Lucey (2013) and Smith et al. (2015). In order to demonstrate that the IMF trend is not caused by the poor data quality of some galaxies, we plot these trends using only the class A subsample in the lower panels of Figure 3, which have the most reliable fitting among the whole sample.

In addition to $\sigma_{\mathrm{e}}$ (see Equation (5)), which approximates the true projected velocity second moment and includes contributions from both ordered rotation $V$ and random motion $\sigma$, we also check whether $\alpha_{\text {IMF }}$ depends on $\sigma$ alone, with the ordered rotation contribution removed. We additionally check the dependence against the specific stellar angular momentum parameter $\lambda_{R_{\mathrm{e}}}$ (Emsellem et al. 2007) and metallicity [Z/H]. Here, $\sigma$ is defined as

$$
\sigma=\sqrt{\left\langle\sigma_{\operatorname{los}}^{2}\left(<R_{\mathrm{e}}\right)\right\rangle}
$$

which removes the velocity term in $\sigma_{\mathrm{e}}$. The results are shown in Figure 4. The IMF trend is similar after changing $\sigma_{\mathrm{e}}$ to $\sigma$. For
$\lambda_{R_{\mathrm{e}}}$ and metallicity $[\mathrm{Z} / \mathrm{H}]$, there is no significant correlation with $\alpha_{\text {IMF. }}$ This is similar to the results from McDermid et al. (2014), who found no strong correlation between IMF and metallicity using the same JAM modeling method. MartínNavarro et al. (2015), however, using line indices, showed that there is a correlation. This could be due to differences between the two methods.

More results about dark matter fractions and the fundamental plane, stellar mass plane, and mass plane scaling relationships will be given in a subsequent paper (H. Li et al. 2017, in preparation).

\subsection{Effects of the Stellar Mass-to-light Ratio Gradient}

For simplicity, when constructing mass models in JAM, a constant stellar mass-to-light ratio is usually assumed in order to convert a luminosity distribution to a stellar mass distribution, such as in Cappellari et al. (2013b). Only recently have dynamical models started to include spatial variations in the stellar mass-to-light ratio, in addition to allowing for a dark matter component (Poci et al. 2016; Mitzkus et al. 2017). This can be important, as a galaxy's stellar population may not be spatially uniform, so there may be a stellar mass-to-light ratio gradient, especially for younger galaxies (Portinari \& Salucci 2010; Tortora et al. 2011; J. Ge et al. 2017, in preparation). In addition, different dust extinction levels at different radii may also affect the mass-to-light ratio profile. It is important therefore to examine the effects caused by mass-to-light ratio gradients.

In order to test the effects of such a gradient, we use the stellar mass profile directly in our mass models instead of the luminosity profile. In doing so, we avoid the assumption of a constant stellar mass-to-light ratio. The stellar mass profile is determined using our full spectrum fitting approach from the MaNGA spectra as described in Section 2.2. The spectra and kinematics are, by design, available over the same region. This is the spatial region where the models are fitted and consequently is the region for which we can constrain the density profiles. The results of dynamical models are only weakly dependent on the adopted stellar mass profiles at larger radii (Krajnović et al. 2005). For this reason, the determination of accurate stellar mass profiles only really matters within the MaNGA field of view. However, to avoid an abrupt and unphysical discontinuity in the stellar mass profile outside the edge of the MaNGA field of view, we use the more approximate color $-M^{*} / L$ relation to smoothly extend the profile out to larger radii.

In practice, to estimate the stellar mass density for our models, we start from the r-band image and multiply the surface brightness of each pixel contained within the MaNGA field of view by the stellar mass-to-light ratios measured from spectral fitting to obtain a stellar mass surface density map. At larger radii we estimate the stellar mass-to-light ratios from the galaxy's color. We take the SDSS g-band and i-band images (Gunn et al. 2006; Eisenstein et al. 2011) and calculate the $g-i$ color in each pixel. We apply the color $-M^{*} / L$ relationship from Bell et al. (2003) to convert the color to the stellar mass-to-light ratio. We assume a Salpeter IMF in the conversion. We use a median filter with window size 9 by 9 pixels (empirically chosen) to obtain a smoothed map. We scale the normalization of the outer part to match the $\left(M^{*} / L\right)_{\text {SPS }}$ values of the inner part around $1 \mathrm{Re}$. This is to 

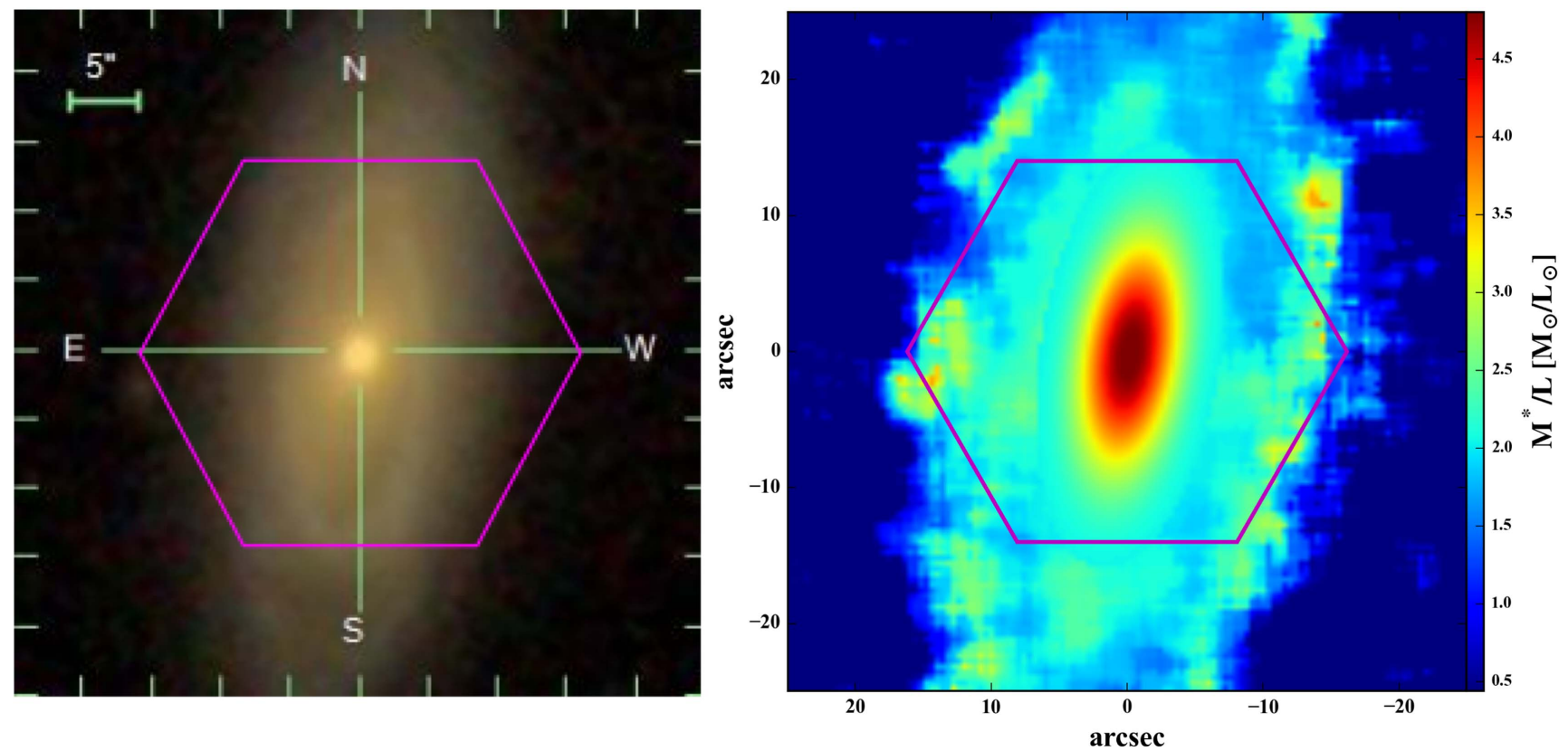

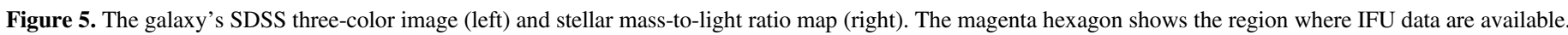
The inner stellar mass-to-light ratios are from MaNGA spectra, and the outer are estimated by the color map.

avoid a discontinuity in the profile, although the scale factor is near 1 for the majority of the galaxies. After obtaining the stellar mass surface density map, we perform the MGE fitting to it to obtain a stellar mass MGE. We then use this stellar mass MGE in our mass model (the luminous MGE is still used as tracer density in JAM modeling). Since we already use stellar mass distribution in the mass model, the scale factor parameter in JAM is not $M^{*} / L$ any more, but the mismatch parameter $\alpha_{\mathrm{IMF}}$ instead.

In Figure 5, we show one galaxy as an example (MaNGA plate-IFU design: 8313-12705), which has one of the largest stellar mass-to-light ratio gradients in our galaxy sample. As can be seen from the color map in the right panel of Figure 5, the stellar mass-to-light ratio at the galaxy center is $\sim 5.0$, decreasing to $\sim 2.0$ in the outer regions.

We take the Class A subset of galaxies and rerun JAM modeling with the gradient correction applied. In Figure 6, we plot the IMF mismatch parameter $\alpha_{\mathrm{IMF}}$ versus $\log \sigma_{\mathrm{e}}$ as in Figure 3 for these galaxies. As can be seen in the figure, even though there is some scatter after applying the gradient correction, the systematic variation still exists, and in fact it becomes even stronger.

In Figure 7, we plot the change of mismatch parameter $\left(\Delta \log \alpha_{\mathrm{IMF}}=\log \alpha_{\mathrm{IMF}}^{\text {corrected }}-\log \alpha_{\mathrm{IMF}}^{\text {uncorrected }}\right) \quad$ versus $\quad \Delta_{M^{*} / L}$ and $\log \sigma_{\mathrm{e}}$. Here, $\Delta_{M^{*} / L}$ is the stellar mass-to-light ratio gradient obtained by fitting the linear function $\log M^{*} / L=a+b \log R$ to the MaNGA IFU results. As can be seen in the top panel, the change in the mismatch parameter increases as the gradient becomes larger (more negative). When the gradient is close to 0 , the $\alpha_{\mathrm{IMF}}$ before and after correction has no systematic difference. However, when the gradient increases, $\alpha_{\mathrm{IMF}}$ systematically decreases after gradient correction (implying lighter IMFs). In the lower panel, one can see that galaxies with lower velocity dispersions have systematically smaller $\alpha_{\mathrm{IMF}}$ after the gradient correction (this means the systematic IMF trend becomes stronger after correcting for the gradient effect). This is because lower-dispersion galaxies have younger stellar populations and steeper gradients. Our test

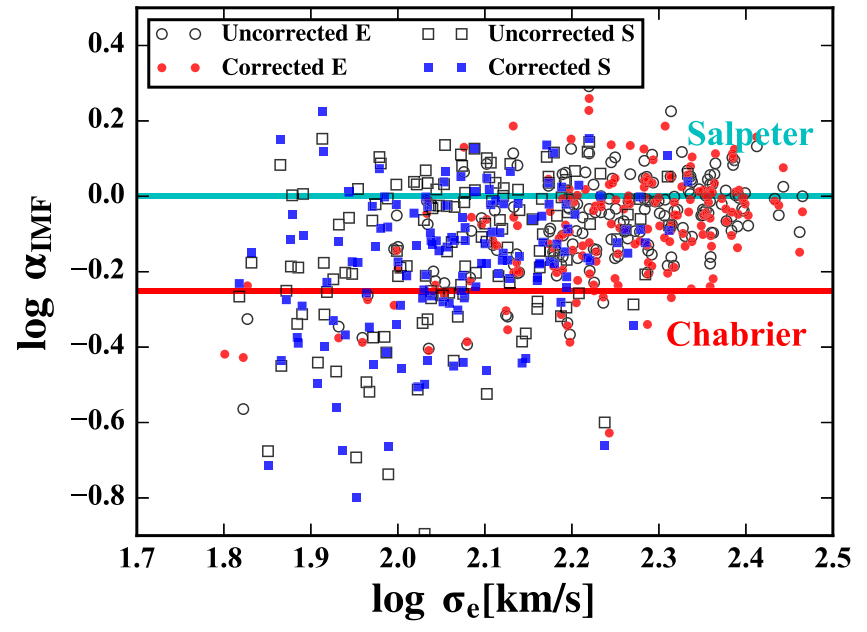

Figure 6. $\log \alpha_{\mathrm{IMF}}$ vs. $\log \sigma_{\mathrm{e}}$ for class A galaxies with the stellar mass-to-light ratio gradient correction applied. The black open circles and squares show the positions of the galaxies without correction for elliptical and spiral galaxies, respectively, and the solid red circles and blue squares show the positions after correction. Other labels and legends are the same as in Figure 3.

suggests that the IMF trend is even stronger than what we determined for the case without gradients as well as in previously published studies.

\subsection{Effect of Inclination and Dust Extinction for Spiral Galaxies}

In this section, we discuss the effects of inclination and dust extinction on spiral galaxies. Since observations suffer more from dust extinction when galaxies are nearly edge-on, the dynamical $M / L$ of flat galaxies tends to be overestimated when galaxies are nearly face-on (Lablanche et al. 2012).

In the top and middle panels of Figure 8 , we show $\log \alpha_{\mathrm{IMF}}$ versus $\log \sigma_{\mathrm{e}}$ for all of the spiral galaxies in our sample with different observed axis ratios (i.e., inclinations) and dust extinction. As can be seen, edge-on galaxies or galaxies with 

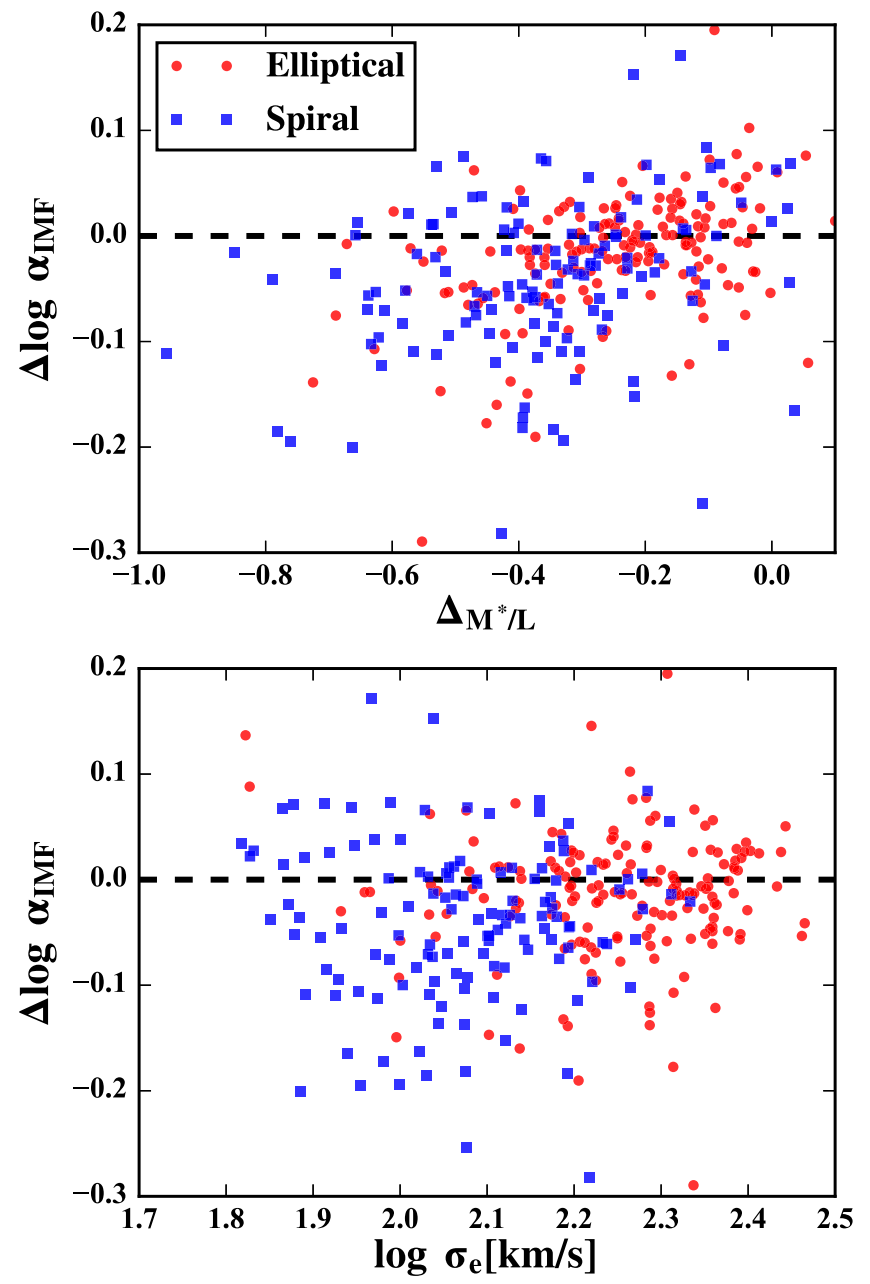

Figure 7. Change of the mismatch parameter $\left(\Delta \log \alpha_{\mathrm{IMF}}=\log \alpha_{\mathrm{IMF}}^{\text {corrected }}\right.$ $\left.\log \alpha_{\mathrm{IMF}}^{\text {uncorrected }}\right)$ vs. gradient (upper) and velocity dispersion (lower). The red circles are elliptical galaxies, and blue squares are spiral galaxies.

higher dust extinction are slightly biased to higher $\alpha_{\text {IMF. This }}$ may be because SPS underestimates the dust extinction or the age of these galaxies, which leads to a lower $\left(M^{*} / L\right)_{\text {SPS }}$. For intermediate and low inclination/dust extinction galaxies, there is no systematic difference. In the bottom panel of Figure 8, we show the value of extinction predicted by our STARLIGHT SPS fits versus axis ratio (i.e., inclination). As expected, edgeon galaxies have more dust extinction, and our trend is similar to the results using optical and near-infrared photometry data obtained by Devour \& Bell (2016). In Figure 9, we further compare the $M^{*} / L_{\text {SPS }}$ assuming a CAL extinction law in SPS with the $M^{*} / L_{\text {SPS }}$ assuming a CCM (Cardelli et al. 1989) extinction law and find no significant difference.

\section{Conclusions}

We have performed JAM modeling for 816 galaxies, with good data quality, from the MaNGA DR13 sample, including both elliptical and spiral galaxies. We have compared the stellar mass-to-light ratios from SPS and JAM modeling, and we find a systematic variation of the IMF for both elliptical and spiral galaxies. Galaxies with lower velocity dispersions within an effective radius are consistent with a Chabrier-like IMF, while galaxies with higher velocity dispersions are consistent with a
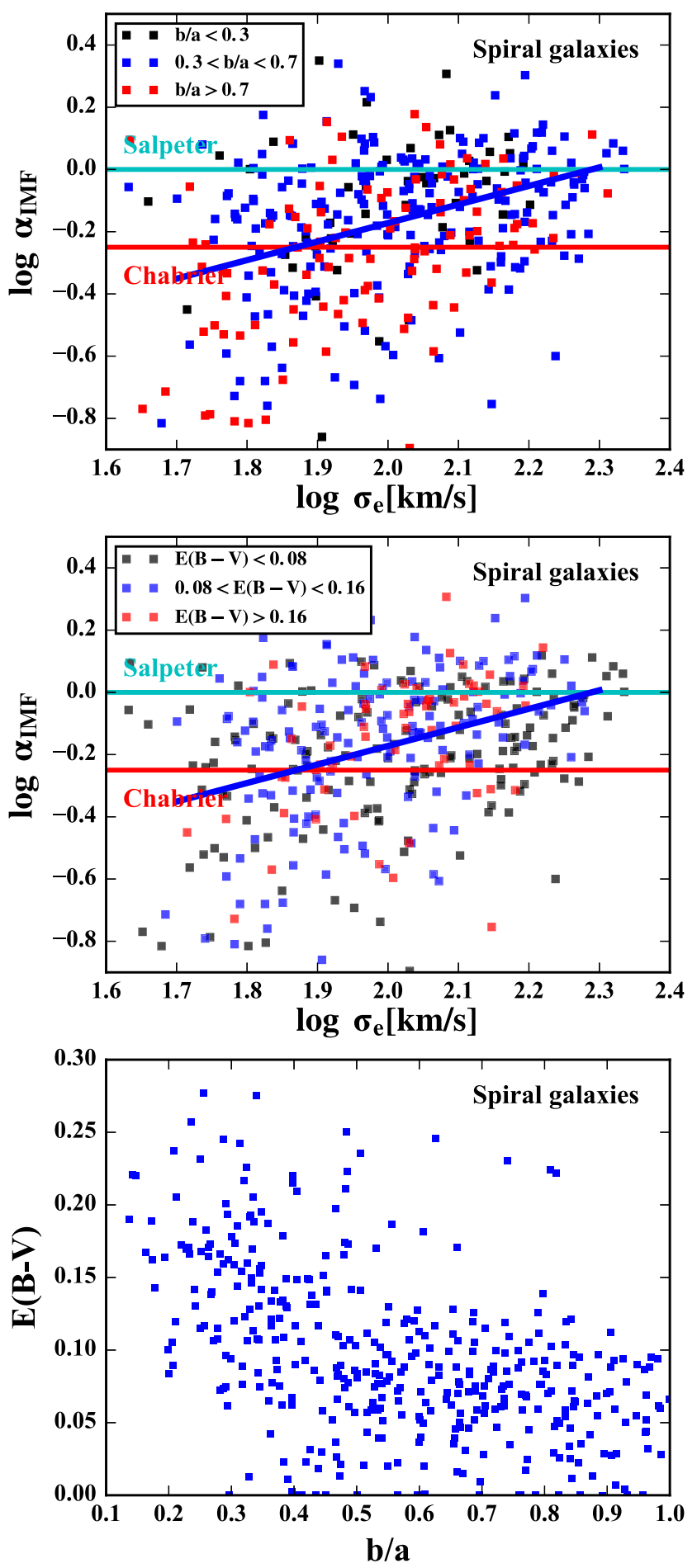

Figure 8. Top: $\log \alpha_{\mathrm{IMF}}$ vs. $\log \sigma_{\mathrm{e}}$ for all of the spiral galaxies with different observed axis ratios. The black squares are for the near edge-on sample $(b / a<0.3)$, blue squares for the intermediate inclined sample $(0.3<b / a<0.7)$, and red squares for the near face-on sample $(b / a>0.7)$. The blue solid line shows the fitting results for spiral galaxies in Table 1. Middle: $\log \alpha_{\mathrm{IMF}}$ vs. $\log \sigma_{\mathrm{e}}$ for all of the spiral galaxies with different extinction values predicted by SPS. The black squares are for the low extinction sample $(E(B-V)<0.08)$, blue squares for the intermediate extinction sample $(0.08<E(B-V)<0.16)$, and red squares for the high extinction sample $(E(B-V)>0.16)$. The blue solid line shows the fitting results for spiral galaxies in Table 1. Bottom: dust extinction values predicted by SPS (STARLIGHT) vs. observed axis ratios. Other labels are the same as in Figure 3. 


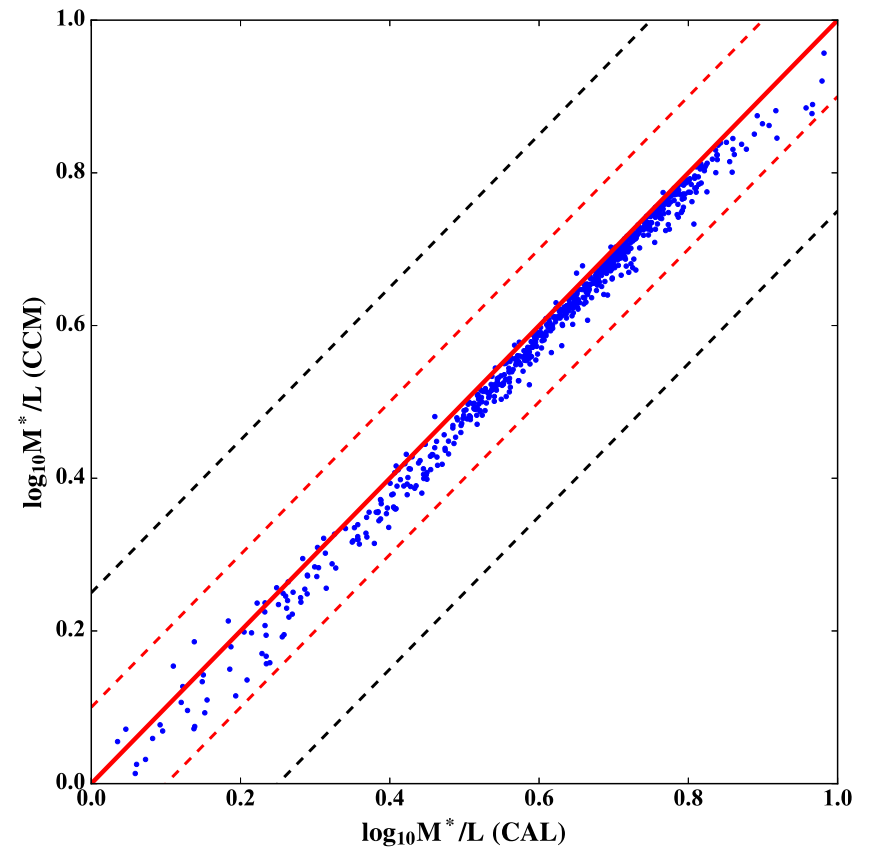

Figure 9. Comparison between the SPS stellar mass-to-light ratios determined using both the CAL and CCM extinction laws. The line colors are the same as in Figure 1.

more bottom-heavy IMF like the Salpeter IMF. These results agree well with previous studies (e.g., Cappellari et al. 2012; Conroy \& van Dokkum 2012; Cappellari et al. 2013a; Dutton et al. 2013a; Tortora et al. 2013; Posacki et al. 2015).

In previous IMF studies using stellar dynamics or gravitational lensing, a constant stellar mass-to-light ratio was assumed. However, there are stellar mass-to-light ratio gradients, especially for young galaxies. So, in addition, we have examined the effect of this gradient. We used our Class A galaxies to introduce this gradient and performed a comparison test. We found that the systematic IMF trend still exists and becomes even stronger after the gradient correction. In addition to the stellar mass-to-light ratio gradient, there are also studies that showed that the IMF inside a galaxy could also be different (Martín-Navarro et al. 2015; La Barbera et al. 2016). In their studies, they found that for several early-type galaxies, the IMF is bottom heavy in the central region, but bottom light in the outer region. This will lead to an even steeper stellar mass-to-light ratio gradient and have some effects on our results. However, the systematic IMF trend in this work is based on a globally averaged IMF for a galaxy. The IMF variation inside a galaxy is beyond the scope of this paper, but we will return to the issue in a future work.

Spiral galaxies with lower velocity dispersions have results with large scatters. This is because they are more affected by cold gas and dust extinction. The degeneracy between dark matter and stellar mass is also stronger in these galaxies. Our results show that these galaxies favor a Chabrier-like IMF, and this is consistent with the results in Bershady et al. (2011), Dutton et al. (2011), and Brewer et al. (2012). Galaxy inclinations do not have strong effects except for nearly edge-on galaxies (higher dust extinction leads to a larger uncertainty in SPS).

Observationally it will be interesting to examine further whether there are differences in the IMF between galaxy disks and bulges in spiral galaxies (e.g., Dutton et al. 2013b). Theoretically it is unclear how the IMF changes when two galaxies with different IMFs merge, and whether the IMF variation changes as a function of redshift. If it does, how this changes the stellar mass function of galaxies and the evolution of the stellar mass as a function of cosmic time needs to be investigated.

H.L. would like to thank Drs. Yiping Shu and Zheng Zheng for many useful discussions and Drs. Anne Jaskot and David Stark for advice on the gas fraction for spiral galaxies. M.C. acknowledges support from a Royal Society University Research Fellowship. We performed our computer runs on the Zen high-performance computer cluster of the National Astronomical Observatories, Chinese Academy of Sciences (NAOC). This work has been supported by the Strategic Priority Research Program "The Emergence of Cosmological Structures" of the Chinese Academy of Sciences Grant No. XDB09000000 (R.J.L. and S.M.), and by the National Natural Science Foundation of China (NSFC) under grant numbers 11333003, 11390372 (S.M.), and 11303033, 11511130054, 11333001 (R.L.), by the Newton Fund, by the Youth Innovation Promotion Association of CAS, and by the World Premier International Research Center Initiative (WPI Initiative), MEXT, Japan.

Funding for the Sloan Digital Sky Survey IV has been provided by the Alfred P. Sloan Foundation, the U.S. Department of Energy Office of Science, and the Participating Institutions. SDSS-IV acknowledges support and resources from the Center for High-Performance Computing at the University of Utah. The SDSS website is www.sdss.org.

SDSS-IV is managed by the Astrophysical Research Consortium for the Participating Institutions of the SDSS Collaboration including the Brazilian Participation Group, the Carnegie Institution for Science, Carnegie Mellon University, the Chilean Participation Group, the French Participation Group, Harvard-Smithsonian Center for Astrophysics, Instituto de Astrofísica de Canarias, The Johns Hopkins University, Kavli Institute for the Physics and Mathematics of the Universe (IPMU)/University of Tokyo, Lawrence Berkeley National Laboratory, Leibniz Institut für Astrophysik Potsdam (AIP), Max-Planck-Institut für Astronomie (MPIA Heidelberg), MaxPlanck-Institut für Astrophysik (MPA Garching), Max-PlanckInstitut für Extraterrestrische Physik (MPE), National Astronomical Observatories of China, New Mexico State University, New York University, University of Notre Dame, Observatário Nacional/MCTI, The Ohio State University, Pennsylvania State University, Shanghai Astronomical Observatory, United Kingdom Participation Group, Universidad Nacional Autónoma de México, University of Arizona, University of Colorado Boulder, University of Oxford, University of Portsmouth, University of Utah, University of Virginia, University of Washington, University of Wisconsin, Vanderbilt University, and Yale University.

\section{References}

Barnabè, M., Dutton, A. A., Marshall, P. J., et al. 2012, MNRAS, 423, 1073 Bastian, N., Covey, K. R., \& Meyer, M. R. 2010, ARA\&A, 48, 339

Bell, E. F., McIntosh, D. H., Katz, N., \& Weinberg, M. D. 2003, ApJS, 149,289

Bershady, M. A., Martinsson, T. P. K., Verheijen, M. A. W., et al. 2011, ApJL, 739, L47

Bigiel, F., \& Blitz, L. 2012, ApJ, 756, 183

Brewer, B. J., Dutton, A. A., Treu, T., et al. 2012, MNRAS, 422, 3574

Bruzual, G., \& Charlot, S. 2003, MNRAS, 344, 1000 
Bryant, J. J., Owers, M. S., Robotham, A. S. G., et al. 2015, MNRAS, 447, 2857

Bundy, K., Bershady, M. A., Law, D. R., et al. 2015, ApJ, 798, 7

Calzetti, D., Armus, L., Bohlin, R. C., et al. 2000, ApJ, 533, 682

Cappellari, M. 2002, MNRAS, 333, 400

Cappellari, M. 2008, MNRAS, 390, 71

Cappellari, M. 2016, ARA\&A, 54, 597

Cappellari, M. 2017, MNRAS, 466, 798

Cappellari, M., Bacon, R., Bureau, M., et al. 2006, MNRAS, 366, 1126

Cappellari, M., \& Copin, Y. 2003, MNRAS, 342, 345

Cappellari, M., \& Emsellem, E. 2004, PASP, 116, 138

Cappellari, M., McDermid, R. M., Alatalo, K., et al. 2012, Natur, 484, 485

Cappellari, M., McDermid, R. M., Alatalo, K., et al. 2013a, MNRAS, 432, 1862

Cappellari, M., Scott, N., Alatalo, K., et al. 2013b, MNRAS, 432, 1709

Cardelli, J. A., Clayton, G. C., \& Mathis, J. S. 1989, ApJ, 345, 245

Chabrier, G. 2003, PASP, 115, 763

Cid Fernandes, R., Mateus, A., Sodré, L., Stasińska, G., \& Gomes, J. M. 2005, MNRAS, 358, 363

Combes, F., García-Burillo, S., Braine, J., et al. 2013, A\&A, 550, A41

Conroy, C., \& van Dokkum, P. G. 2012, ApJ, 760, 71

Devour, B. M., \& Bell, E. F. 2016, MNRAS, 459, 2054

Drory, N., MacDonald, N., Bershady, M. A., et al. 2015, AJ, 149, 77

Dutton, A. A., Conroy, C, van den Bosch, F. C., et al. 2011, MNRAS, 416, 322

Dutton, A. A., Macciò, A. V., Mendel, J. T., \& Simard, L. 2013a, MNRAS, 432, 2496

Dutton, A. A., Macciò, A. V., Stinson, G. S., et al. 2015, MNRAS, 453, 2447

Dutton, A. A., Treu, T., Brewer, B. J., et al. 2013b, MNRAS, 428, 3183

Eisenstein, D. J., Weinberg, D. H., Agol, E., et al. 2011, AJ, 142, 72

Emsellem, E., Cappellari, M., Krajnović, D., et al. 2007, MNRAS, 379, 401

Emsellem, E., Monnet, G., \& Bacon, R. 1994, A\&A, 285, 723

Foreman-Mackey, D., Hogg, D. W., Lang, D., \& Goodman, J. 2013, PASP, 125,306

Gunn, J. E., Siegmund, W. A., Mannery, E. J., et al. 2006, AJ, 131, 2332

Hogg, D. W., Baldry, I. K., Blanton, M. R., \& Eisenstein, D. J. 2002, arXiv: astro-ph/0210394

Huang, S., Haynes, M. P., Giovanelli, R., \& Brinchmann, J. 2012, ApJ, 756,113

Jaskot, A. E., Oey, M. S., Salzer, J. J., et al. 2015, ApJ, 808, 66

Krajnović, D., Cappellari, M., Emsellem, E., McDermid, R. M., \& de Zeeuw, P. T. 2005, MNRAS, 357, 1113

La Barbera, F., Vazdekis, A., Ferreras, I., et al. 2016, MNRAS, 457, 1468
Lablanche, P.-Y., Cappellari, M., Emsellem, E., et al. 2012, MNRAS, 424, 1495

Law, D. R., Cherinka, B., Yan, R., et al. 2016, AJ, 152, 83

Law, D. R., Yan, R., Bershady, M. A., et al. 2015, AJ, 150, 19

Li, H., Li, R., Mao, S., et al. 2016, MNRAS, 455, 3680

Lintott, C. J., Schawinski, K., Slosar, A., et al. 2008, MNRAS, 389, 1179

Martín-Navarro, I., Barbera, F. L., Vazdekis, A., Falcón-Barroso, J., \& Ferreras, I. 2015, MNRAS, 447, 1033

McDermid, R. M., Cappellari, M., Alatalo, K., et al. 2014, ApJL, 792, L37

Mitzkus, M., Cappellari, M., \& Walcher, C. J. 2017, MNRAS, 464, 4789

Panter, B., Jimenez, R., Heavens, A. F., \& Charlot, S. 2007, MNRAS, 378,1550

Poci, A., Cappellari, M., \& McDermid, R. M. 2016, arXiv:1612.05805

Portinari, L., \& Salucci, P. 2010, A\&A, 521, A82

Posacki, S., Cappellari, M., Treu, T., Pellegrini, S., \& Ciotti, L. 2015, MNRAS, 446, 493

Salpeter, E. E. 1955, ApJ, 121, 161

Sánchez, S. F., Kennicutt, R. C., Gil de Paz, A., et al. 2012, A\&A, 538, A8

Sánchez-Blázquez, P., Peletier, R. F., Jiménez-Vicente, J., et al. 2006, MNRAS, 371, 703

Schaller, M., Frenk, C. S., Bower, R. G., et al. 2015, MNRAS, 451, 1247

SDSS Collaboration, Albareti, F. D., Allende Prieto, C., et al. 2016, arXiv: 1608.02013

Smee, S. A., Gunn, J. E., Uomoto, A., et al. 2013, AJ, 146, 32

Smith, R. J., Alton, P., Lucey, J. R., Conroy, C., \& Carter, D. 2015, MNRAS, 454, L71

Smith, R. J., \& Lucey, J. R. 2013, MNRAS, 434, 1964

Spiniello, C., Trager, S. C., Koopmans, L. V. E., \& Chen, Y. P. 2012, ApJL, 753, L32

Tollet, E., Macciò, A. V., Dutton, A. A., et al. 2016, MNRAS, 456, 3542

Tortora, C., Napolitano, N. R., Romanowsky, A. J., et al. 2011, MNRAS, 418, 1557

Tortora, C., Napolitano, N. R., Romanowsky, A. J., Capaccioli, M., \& Covone, G. 2009, MNRAS, 396, 1132

Tortora, C., Romanowsky, A. J., \& Napolitano, N. R. 2013, ApJ, 765, 8

Treu, T., Auger, M. W., Koopmans, L. V. E, et al. 2013, ApJ, 709, 1195

Vazdekis, A., Sánchez-Blázquez, P., Falcón-Barroso, J., et al. 2010, MNRAS, 404, 1639

Yan, R., Bundy, K., Law, D. R., et al. 2016b, AJ, 152, 197

Yan, R., Tremonti, C., Bershady, M. A., et al. 2016a, AJ, 151, 8 